\title{
Acknowledgement to Reviewers of the International Journal of Financial Studies in 2015
}

\author{
International Journal of Financial Studies Editorial Office, \\ Published: 22 January 2016 \\ MDPI AG, Klybeckstrasse 64, CH-4057 Basel, Switzerland; ijfs@mdpi.com
}

The editors of the International Journal of Financial Studies Editorial Office, would like to express their sincere gratitude to the following reviewers for assessing manuscripts in 2015.

We greatly appreciate the contribution of expert reviewers, which is crucial to the journal's editorial decision-making process. Several steps have been taken in 2015 to thank and acknowledge reviewers. Good, timely reviews are rewarded with a discount off their next MDPI publication. By creating an account on the submission system, reviewers can access details of their past reviews, see the comments of other reviewers, and download a letter of acknowledgement for their records. This is all done, of course, within the constraints of reviewer confidentiality. Feedback from reviewers shows that most see their task as a voluntary and mostly unseen work in service to the scientific community. We are grateful to our reviewers for the contribution they make.

Agliardi, Elettra

Andrikopoulos, Panagiotis

Antzoulatos, Angelos A.

Ararat, Melsa

Bahmani, Sahar

Balvers, Ronald

Barnett, William

Barro, Diana

Basse, Tobias

Bazzana, Flavio

Belomestny, Denis

Bhaumik, Sumon

Bo, Hong

Borowski, Krzysztof

Cakmakli, Cem

Cesaratto, Sergio

Choudhry, Taufiq

Ćurak, Marijana

Danuletiu, Dan Constantin

Eling, Martin

Emery, Douglas R.

Febrero, Eladio

Feng, Zhijian

Ferrero, Giuseppe

Fillat, José L.

Filson, Darren

Fuh, Cheng-Der

Gatfaoui, Hayette

González, Federico

Grechyna, Daryna
Guan, Lim Kian
Guidi, Francesco
Gupta, Rakesh
Hasanov, Mübariz
Hendrickson, Joshua
Hill, James R.
Ho, Shu-Yi
Hovemann, Gregor
Hsu, Ming-Fu
Huang, Wanwan
Iiboshi, Hirokuni
Ireland, Peter N.
Isakov, Dušan
Itzkowitz, Jennifer
Ivanov, Stoyu I.
Jankensgård, Håkan
Johnson, Steve
Jolly, Nicholas
Keddad, Benjamin
Kim, Jintae
Koutmos, Dimitrios
Kozak, Sylwester
Krause, Timothy
Lee, Ming-Chang
Lence, Sergio H.
Leung, Mark T.
Leung, Tim
Lin, Hong-Jen

Grechyna, Daryna

Guan, Lim Kian

Guidi, Francesco

Gupta, Rakesh

Hasanov, Mübariz

Hendrickson, Joshua

Hill, James R.

Ho, Shu-Yi

Hovemann, Gregor

$\mathrm{Hsu}$, Ming-Fu

Huang, Wanwan

Ireland, Peter N.

Isakov, Dušan

Itzkowitz, Jennifer

Ivanov, Stoyu I.

Jankensgård, Håkan

Johnson, Steve

Jolly, Nicholas

Keddad, Benjamin

Kim, Jintae

Koutmos, Dimitrios

Kozak, Sylwester

Krause, Timothy

Lee, Ming-Chang

Lence, Sergio H.

Leung, Tim

Lin, Hong-Jen

Madhavan, Ananth
Maurer, Thomas
Michael-Tsabari, Nava
Michiels, Anneleen
Mori, Naoya
Morley, Bruce
Obizhaeva, Anna
Oh, Kyong Joo
Pan, Zhibin
Papailias, Fotis
Pawlowski, Tim
Pervan, Maja
Powell, Robert J.
Prasad, Dev
Ratti, Ronald
Riddiough, Steven
Riley, Jennifer
Rindi, Barbara
Rockerbie, Duane W.
Safarian, Mher
Samitas, Aristeidis
Sapena, Juan
Seifried, Chad
SenGupta, Indranil
Serletis, Apostolos
Sibilkov, Valeriy
Siddiqui, Javed
Siganos, Antonios
Singer, Ronald F.

Madhavan, Ananth

Maurer, Thomas

Michael-Tsabari, Nava

Michiels, Anneleen

Mori, Naoya

Morley, Bruce

Obizhaeva, Anna

Oh, Kyong Joo

Pan, Zhibin

Papailias, Fotis

Pawlowski, Tim

Pervan, Maja

.

sad, Dev

Ratti, Ronald

Riddiough, Steven

Riley, Jennifer

Rindi, Barbara

Rockerbie, Duane W.

Safarian, Mher

Samitas, Aristeidis

Sapena, Juan

Seifried, Chad

SenGupta, Indranil

Serletis, Apostolos

Sibilkov, Valeriy

Singer, Ronald F. 
Skindilias, Konstantinos Sly, Nicholas

Sundaresh, Harish

Talukdar, Bakhtear

Nguyen Thi, Thanh-Binh

Tong, Tan Hun

Tsoumas, Christos

Viale, Ariel M.

Lozano-Vivas, Ana
Wang, Kuan Min

Watanabe, Nicholas

Weber, William L.

Wu, Jyh-Lin

Xia, Fan Dora

Xie, Xiaoying

Yang, Li

Yang, Xiaolan

Yang, Haijun
Yang, Charles

Yüksel, Aslı

Zagaglia, Paolo

Zakamulin, Valeriy I.

Zedda, Stefano

Zhu, Huiming

Zucchi, Francesca

(C) 2016 by the author; licensee MDPI, Basel, Switzerland. This article is an open access article distributed under the terms and conditions of the Creative Commons by Attribution (CC-BY) license (http://creativecommons.org/licenses/by/4.0/). 